\title{
A Data-Driven Semi-Automatic Framenet Development Methodology
}

\author{
Shafqat Mumtaz Virk \\ Språkbanken Text, Dept. of Swedish \\ University of Gothenburg \\ 40530 Gothenburg, Sweden \\ virk.shafqategmail.com
}

\author{
Lars Borin \\ Språkbanken Text, Dept. of Swedish \\ University of Gothenburg \\ 40530 Gothenburg, Sweden \\ lars.borinesvenska.gu. se
}

\author{
Dana Dannélls \\ Språkbanken Text, Dept. of Swedish \\ University of Gothenburg \\ 40530 Gothenburg, Sweden \\ dana.dannellsesvenska.gu.se
}

\author{
Markus Forsberg \\ Språkbanken Text, Dept. of Swedish \\ University of Gothenburg \\ 40530 Gothenburg, Sweden \\ markus. forsberg@svenska.gu. se
}

\begin{abstract}
FrameNet is a lexical semantic resource based on the linguistic theory of frame semantics. A number of framenet development strategies have been reported previously and all of them involve exploration of corpora and a fair amount of manual work. Despite previous efforts, there does not exist a well-thought-out automatic/semi-automatic methodology for frame construction. In this paper we propose a data-driven methodology for identification and semi-automatic construction of frames. As a proof of concept, we report on our initial attempts to build a widerscale framenet for the legal domain (LawFN) using the proposed methodology. The constructed frames are stored in a lexical database and together with the annotated example sentences they have been made available through a web interface.
\end{abstract}

\section{Introduction}

Frame semantics is a theory of meaning in natural languages proposed by Charles Fillmore (Fillmore, 1977, 1982). The theory stipulates that meanings of words can be best understood with reference to the situations they invoke in the minds of the speakers. The concrete manifestation of frame semantics is the computational lexical resource FrameNet, first constructed within the English Berkeley FrameNet (BNF) project (Baker et al., 1998). The resource has inspired work on framenets for many other languages and domains, including Chinese (You et al., 2007), French (Candito et al., 2014), German (Burchardt et al., 2009), Hebrew (Hayoun and Elhadad, 2016), Korean (Kim et al., 2016), Italian (Lenci et al., 2010), Japanese (Saito et al., 2008), Portuguese (Torrent, 2013), Spanish (Subirats, 2009), and Swedish (Borin et al., 2010a; Dannélls et al., Forthc.a).

FrameNet is built around the notion of frames. A frame in this context is a schematic representations of events, objects, situations, institutions, etc. The primary components of a frame are the frame definition along with the frame elements and the lexical units documented with corpus evidence (Ruppenhofer et al., 2016).

There are at least three major framenet development strategies that have been reported in the literature (Candito et al., 2014) namely: (1) lexicographic frame-by-frame (2) corpus driven lemmaby-lemma (3) the full-text strategy. Of these three, the most commonly applied strategy is frame-byframe, where the frame is defined along with its frame-elements first. Example sentences are then chosen from a corpus for a disambiguated annotation of lexical units and frame-elements. In the lemma-by-lemma strategy, a set of lemmas (lexical units) are chosen first and then all their occurrences are annotated in a given corpus together with the annotation of frame-elements. In the full-text strategy, all content words in a given text are annotated. The latter two strategies pre-suppose that frames already exist, although new frames can also be developed as new senses of lexical units are encountered in the text.

All three strategies involve exploration of corpora and a fair amount of manual work for construction of semantic frames, and for searching suitable example sentences to be annotated. Various framenet development projects have reported the use of (or developed) tools to assist in the corpus exploration and frames construction parts (e.g. the Swedish FrameNet++ project; Borin et al., 2010b; Dannélls et al., Forthc.b), and there also exist guide- 
lines for frame construction (Burchardt et al., 2009; Ruppenhofer et al., 2016). However, there is no well-thought-out automatic/semi-automatic procedure for frame development from scratch.

In this study, we propose a data-driven method for the identification and semi-automatic construction of domain-specific semantic frames. The identification involves recognizing the domain-specific events, entities, relations, procedures, etc. for which the semantic frames can be constructed. The development part involves discovering various semantic roles of a given frame, spotting and linking lexical units to the target frame, and annotating example sentences.

\section{Data and Pre-processing}

As a proof of concept of our methodology, we prepared a small data-set by collecting a set of documents from the legal domain and applied OCR processing to produce a machine readable version. The data was then enriched with metadata and linguistic annotations. It was then stored and made accessible through a corpus infrastructure tool for easy excess and exploration.

The data used in this study was downloaded from web repository of the united nations high commissioner for refugees. ${ }^{1}$ A subset of 900 pdf documents resulting from the hits for the search string "well-founded fear of being persecuted"2 were downloaded and then OCRed using ABBYY FineReader software. ${ }^{3}$ Each document was processed for the following document, token, and structure level attributes.

- Text-Level Attributes: Article title, author, publisher, topic, and country.

- Token-Level Attributes: word tokenization, lemmatization and part of speech (POS) tags.

- Structure-Level: Sentence segmentation and dependency parses.

Document level attributes were preserved while downloading the documents, and for various token and structure level annotations we used Sparv (Borin et al., 2016), which is an annotation pipeline

\footnotetext{
${ }^{1}$ wWw.refworld.org

${ }^{2}$ This search string was chosen in connection to another part of the project with its own objectives of analyzing the ill-treatment of refugees.

${ }^{3}$ https : / / www . abbyy . com/
}

developed and maintained at Språkbanken Text. ${ }^{4}$ It can be used to automatically annotate textual data with various token, text, and structure level attributes using in-house and third party annotation tools. We used Stanford's NLP toolkit (Manning et al., 2014) for token and structure level annotations. After annotating the data, it was made available through Korp (Borin et al., 2012), a stateof-the-art corpus infrastructure tool developed and maintained by Språkbanken Text. It provides various basic and advanced level features to better search, explore, and visualize a corpus. Figure 1 shows a screenshot of the search results together with various annotations when searched for a simple string 'article'. The left-hand pane shows the sentences containing the search string, and the right-hand pane shows various text level (title, author, topic, etc.) and token level (lemma, pos-tag, etc.) tags for the selected token (the word 'title' highlighted with black background). The bottom right corner also shows the dependency parse tree of the sentence.

The extended search tab provides options to search for any of the individual attributes (e.g. author, title, pos-tag etc.) or a combination of them combined by and/or logical operators. The advanced tab allows the user to formulate a search query using the CQP query language (Christ, 1994). The data is password protected due to IPR issues and is intended to be used for internal research purposes at this stage. However, in the future we have plans to release it with suitable licensing options.

\section{Frame Development Methodology}

A step-wise description of the frame development methodology is given below. This is followed by construction of an example frame using the described methodology. The developed frame is a part of the legal domain framenet (more details about the legal domain framenet in Section 4).

1. As a first step, the word segmented data reported in Section 2 is sorted in the descending order of term ${ }^{5}$ frequencies after removing the function words. The purpose is to identify the

\footnotetext{
${ }^{4}$ Språkbanken Text is a research unit and also forms part of Nationella språkbanken (the National Language Bank), a national e-infrastructure supporting research based on language data in Sweden.

${ }^{5}$ Here, we use "term" in a basically non-technical sense, to mean '(text word) type' or 'lemma'.
} 


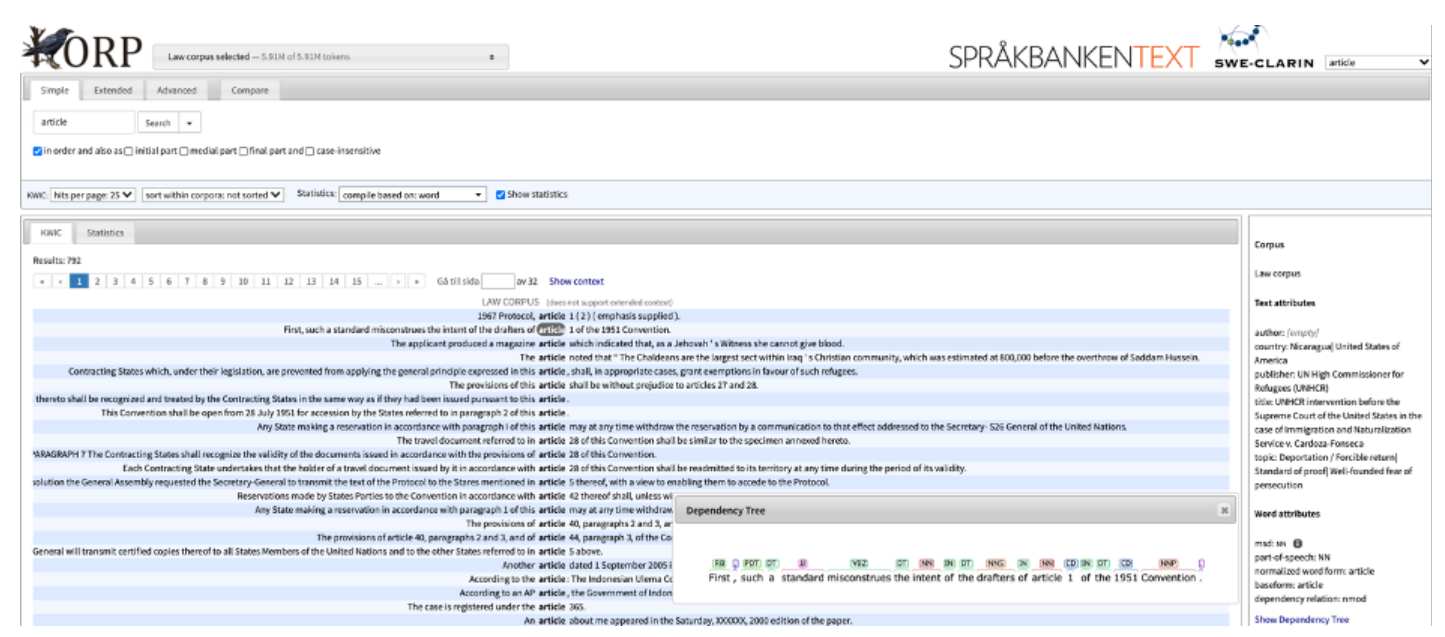

Figure 1: A Korp screenshot showing search hits for the search string 'article'

most frequently used terms and cover them first while designing the frames.

2. Each item in the obtained list is inspected manually to decide if it is a domain-specific term or not with the help of a domain expert if required. In a better setting, the word list can be filtered out beforehand with the help of any already existing domain-specific terminology list or a lexical database such as WordNet (Fellbaum, 1998) to speed up the process and reduce the manual work, but we leave this aspect to be explored in the future.

3. Once a term has been determined to be a domain-specific term, the next step is to check if the term fits as a lexical unit of an already existing frame or not. If it does, it is simply added as a lexical unit of the existing frame. If not, it means a new frame needs to be designed. A suitable name for the frame is chosen, and the following procedure is followed to identify various frame-elements of the frame. Let the term be $\mathrm{T}$.

(a) All sentences containing the term $\mathrm{T}$ are extracted from the data and their dependency parses are retrieved from the parsed data reported in Section 2.

(b) From the dependency parses, the head and dependent text segments are grouped together for each of the dependency relations.

(c) The text segments in each group are then manually inspected and a decision is made to whether we need a frame ele- ment or not. ${ }^{6}$ If yes, a suitable frameelement title is chosen, and the element is made part of the frame. This step is repeated for each dependency relation.

Lets now walk through the construction of an example frame Article using the above described methodology. In our data collection, the term 'article' occurred 29 times, and it is easy to recognize that this term has a legal domain sense in addition to four others as per WordNet. Considering that no frames have been designed previously, we need to construct a frame for which the word 'article' will be a lexical unit. This is step 2 of our methodology. We chose the name Article for the frame, and move towards construction of its structure i.e. step 3. Table 1 shows text segments generated using step 3 for the syntactic relations 'dobj' (i.e. direct object) ${ }^{7}$ and 'nmod' (nominal modifier). For space reasons, only a few entries for a selected set of relations are shown.

A manual inspection of the text segments under the 'Dependent' for the 'dobj' relation reveals that the word 'article' whenever used in the legal domain is often followed

\footnotetext{
${ }^{6}$ This is basically a linguistic decision which requires both experience of framenet development and training in grammatical and lexical-semantic analysis for the language in question ([legal] English in our case). See Ruppenhofer et al. (2016) for a more detailed discussion of the considerations and reasoning involved.

${ }^{7}$ The constituents in question are not direct objects; rather, the relation should be labeled 'flat' or possibly 'appos' under the UD scheme. This does not matter to our example, but it will of course become important when we will be looking to automate the discovery of frame elements further.
} 
by a figure or figures that refers to the article number of the constitution/agreement. This means we need a frame-element to capture and represent this usage. It can perhaps be argued that it is so obvious that the term 'article' will be followed by an article number so why we need to follow the described procedure. It may appear obvious in this case, but it can not be generalized across frames and frame-elements (imagine the construction of the frame Injunction which refers to a judicial order restraining a person from beginning or continuing an action). Our experience suggests that this usage based methodology is very helpful in identifying the frames and frame-elements.

Similarly, a manual inspection of the text segments under the 'Dependent' for 'nmod' relation revealed that there is often a mention of a law/protocol/constitution to which the article belongs e.g 'of the Turkish constitution'. This means we need a frame-element to capture that information, hence, the frame-element Constitution. The same procedure was followed to design the frame-elements INTERPRETATION, and DATE while inspecting the 'acl:relcl' (i.e. relative clause modifier) and 'nmod:tmod' (i.e. temporal modifier) relations respectively.

For the actual frame construction and storage, we used Karp, which is another lexical infrastructure tool developed and maintained at Språkbanken. Figure 2 shows a screenshot of the tool and the structure of the Article frame. It also shows a set of annotated examples and the lexical units which can trigger this frame.

\section{A Legal Domain Framenet and its Applications}

\subsection{LawFN}

General-language lexical resources such as BFN are both too broad and too narrow for successful deployment in domain-specific natural language processing (NLP) applications. On the one hand, they contain more than one sense for many headwords, most of which are not relevant in the domain of interest (but which lower the accuracy of the application by introducing irrelevant ambiguity in the analysis). On the other, they often lack some important domain-specific usages. For this reason, several domain-specific framenets have been compiled, covering e.g. medicine, football/soccer and tourism (Borin et al., 2007; Schmidt, 2009; Torrent et al., 2014).

There have been some initiatives reported in literature on building framenets for the legal domain (e.g. Venturi, 2011; Bertoldi and Chishman, 2012). However, to the best of our knowledge, these efforts have been quite limited in scope, and no fullscale resource of the kind proposed here has been presented. In this study, we report on our initial attempts to build a wider-scale FrameNet for the legal domain that we call LawFN.

\subsection{Applications of LawFN}

The major motivation behind initiating the development of LawFN is its potential applications in the area of Legal Tech, which refers to the use of technology and software for providing various legal services and support to the legal industry. In recent years, Legal Tech has gain popularity, and the use of technology is increasing in many legaldomain activities such as case management, contract management, document automation and analysis (Gruzauskas and Ragavan, 2020), etc. Some of these tasks require semantic analysis of the text within legal domain documents.

When we apply currently available semantic analysis technology to extract information about laws automatically from text we do not retrieve the desired analysis. For example when we analyze the sentence: "Justice Kirby similarly stated in the same judgement that the convention does not require or imply the elimination by the state of all risks of harm; rather it posits a reasonable level of protection, not a perfect one.", with the general framework for semantic role labeling (Punyakanok et al., 2008), ${ }^{8}$ we get the analysis as shown in Example 1.

(1) [Justice Kirby $]_{\text {Announcer }}[\text { similarly }]_{\text {Discourse }}$ $[\text { stated }]_{L U}[\text { in the same judgement }]_{\text {Location }}$ [that the convention does not require or imply the elimination by the state of all risks of harm; rather it posits a reasonable level of protection, not a perfect one.] $]_{\text {Utterance }}$

As Example 1 shows, the identified LU is 'state', and the frame elements are ANNOUNCER, DIS-

\footnotetext{
${ }^{8}$ https: / / cogcomp. seas.upenn.edu/page/ demo_view/sr]
} 


\begin{tabular}{|c|c|c|}
\hline \multicolumn{3}{|r|}{ Relation: dobj (i.e. direct object) } \\
\hline Head & Dependent & Sentence \\
\hline 'articles' & '20 and 30' & $\begin{array}{l}\text { 'The recast Directive continues.... "mental health problems" and the Parlia- } \\
\text { ment's "mental health illnesses" under articles } 20 \text { and } 30 \text {.' }\end{array}$ \\
\hline 'articles' & $\begin{array}{l}\text { ' } 6,7 \text { and } 8 \text { of the } \\
\text { Statute' }\end{array}$ & 'Particularly relevant for exclusion are articles 6,7 and 8 of the Statute, .....' \\
\hline 'articles’ & $\begin{array}{l}\text { '2 to } 34 \text { inclusive of } \\
\text { the Convention' }\end{array}$ & $\begin{array}{l}\text { 'The States Parties to the present Protocol undertake to apply articles } 2 \text { to } 34 \\
\text { inclusive of the ...' }\end{array}$ \\
\hline \multicolumn{3}{|r|}{ Relation: nmod (i.e. nominal modifier) } \\
\hline 'articles' & 'of the Convention' & 'With respect to those articles of the Convention to be applied ...Federal States;' \\
\hline 'article' & $\begin{array}{l}\text { 'of the European } \\
\text { Convention on } \mathrm{Hu}- \\
\text { man Rights' }\end{array}$ & $\begin{array}{l}\text { 'They have been given leave to enter the United Kingdom because article } 3 \text { of } \\
\text { the European Convention on Human Rights forbids their return ... punishment } \\
\text { there.' }\end{array}$ \\
\hline 'article' & $\begin{array}{l}\text { 'of the Turkish Con- } \\
\text { stitution.' }\end{array}$ & 'The essential point..... under article 24 of the Turkish Constitution.' \\
\hline \multicolumn{3}{|r|}{ Relation: acl:relcl (i.e. relative clause) } \\
\hline 'article' & $\begin{array}{l}\text { 'which embodies an } \\
\text { immediate obliga- } \\
\text { tion to respect and } \\
\text { ensure all of the rel- } \\
\text { evant rights' }\end{array}$ & $\begin{array}{l}\text { 'In this sense the obligation differs significantly from that contained in article } 2 \\
\text { of the International Covenant on Civil and Political Rights which embodies an } \\
\text { immediate obligation to respect and ensure all of the relevant rights.' }\end{array}$ \\
\hline 'article' & $\begin{array}{l}\text { 'which guarantees } \\
\text { "women equality } \\
\text { with men before the } \\
\text { law' }\end{array}$ & $\begin{array}{l}\text { 'This contravenes the right of women under, article } 15(9) \text { of the Convention on } \\
\text { the Elimination of Discrimination against Women, which guarantees "women } \\
\text { equality with men before the law.' }\end{array}$ \\
\hline
\end{tabular}

Table 1: Frame Elements of the Article Frame

COURSE and UTTERANCE. We can compare this analysis to the one resulting from the legal domain framenet specification in Figure 3. It can be observed there are huge differences between the analyses. First, the LUs that are central to the legal domain, here 'justice' and 'judgement' are captured as frame elements. Second, the frame-element labels are domain independent and hence not suitable for the legal domain. If we want better semantic analysis, amenable to further computational processing based on text semantics, e.g. (logical) inference or comparison of textual sources with regard to their domain specific content rather than their linguistic form, there must be a suitable presentation of the domain in question.

\subsection{Frame Types in LawFN}

For a better organization, we divide frames in the LawFN into two types: Entity frames and Event frames. Entity frames are simpler in their structure and they are meant to represent various legal domain entities e.g. judge, court, tribunal, etc. Event frames are a bit more complex in their structure and are meant to represent various legal domain events or processes such as prosecution, judgement, defense, etc. In another sense, entity frames act as slot fillers for various semantic roles of an event frame. Consider Figure 3 showing an annotated sentence in which a filler frame (i.e. Judge) files in a semantic role of an event frame (i.e. Judgement).

In the annotated sentence, the frame Judge is triggered by the lexical unit 'justice' and NAME is the only frame element which is realized in this sentence (the annotation in red color). This frame then becomes frame-element JUDGE for the semantic frame Judgement triggered by the lexical unit 'judgement'. The Judgement frame is the only other realized frame-element (annotation in green).

\subsection{Current Status of LawFN}

Using the proposed frame-development methodology, we have developed a total of 10 frames, containing 36 frame-elements, 24 lexical units, and 22 annotated example sentences. A list of constructed frames together with their frame-elements, lexical units, and annotated example sentences are provided in Appendix A.

\section{Conclusions and Future Work}

Our contribution is twofold. First, we have reported a semi-automatic frame-development methodology, which can help speed up the frame development process. Second, we have reported initial attempts in building a framenet for the legal domain and annotated data as lexico-semantic resources. 


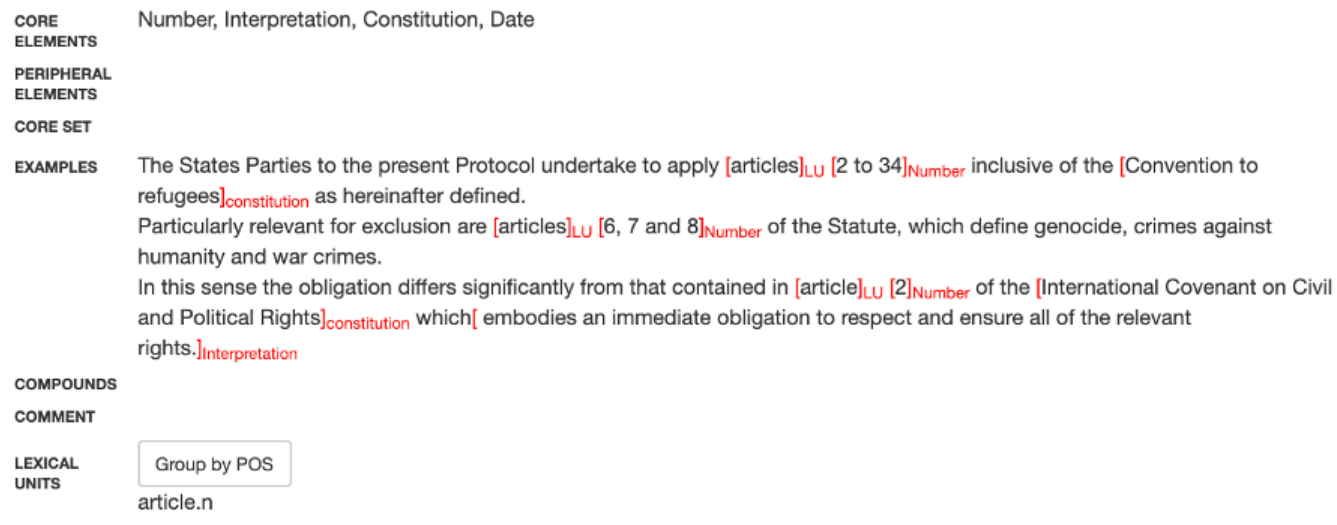

Figure 2: A Karp screenshot showing structure of the Article frame

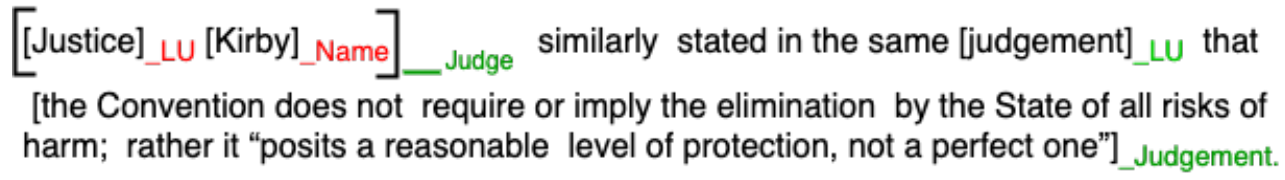

Figure 3: Sentence semantic analysis according to LawFN

In the future, we have plans to continue constructing more frames. Our dataset is quite limited in scope and size, which we plan to extend in the near future. With the bigger data set, we will be able to explore more syntactic patterns. This means capturing more possible use cases, and hence being in a position to better design the frames. Also, we have plans to annotate data with the developed frames, and build a frame-semantic parser exploiting machine learning. The developed parser then can be used for automatic semantic analysis of legal domain documents, which then can be used to extract particular types of information from those document. As an example, suppose one is interested to extract all judgements made by a particular judge under a particular law/protocol/agreement from a corpus of court decisions. Parsing the corpus for Judgement, Law, and Article frames can help extract that information automatically. Evaluation of the extracted information for accuracy is another direction that we plan to explore in the future.

\section{Acknowledgments}

The work described here has been funded in part by the Swedish Research Council and by the University of Gothenburg, through their support of the Swedish national research infrastructure The National Swedish Language Bank (SRC grant
2017-00626) and of Språkbanken Text, and in part by From Dust to Dawn: Multilingual Grammar Extraction from Grammars project funded by Stiftelsen Marcus och Amalia Wallenbergs Minnesfond 2007.0105, Uppsala University;

\section{References}

Collin F. Baker, Charles J. Fillmore, and John B. Lowe. 1998. The Berkeley FrameNet project. In Proceedings of ACL/COLING 1998. ACL, Montreal, pages 86-90. https://doi.org/10.3115/980845.980860.

Anderson Bertoldi and Rove Chishman. 2012. Frame semantics and legal corpora annotation: theoretical and applied challenges 7 .

Lars Borin, Dana Dannélls, Markus Forsberg, Dimitrios Kokkinakis, and Maria Toporowska Gronostaj. 2010a. The past meets the present in Swedish FrameNet++. In Anne Dykstra and Tanneke Schoonheim, editors, Proceedings of the 14th EURALEX International Congress. Fryske Akademy, Leeuwarden/Ljouwert, pages 269-281.

Lars Borin, Dana Dannélls, Markus Forsberg, Maria Toporowska Gronostaj, and Dimitrios Kokkinakis. 2010b. Swedish FrameNet++. In SLTC 2010. Linköping University, Linköping.

Lars Borin, Markus Forsberg, Martin Hammarstedt, Dan Rosén, Roland Schäfer, and Anne Schumacher. 2016. Sparv: Språkbanken's corpus annotation pipeline infrastructure. In SLTC 2016. The Sixth Swedish Language Technology Conference, Umeå University, 17-18 November, 2016. 
Lars Borin, Markus Forsberg, and Johan Roxendal. 2012. Korp - the corpus infrastructure of Språkbanken. In Proceedings of LREC 2012. ELRA, Istanbul, pages 474-478. http://www.lrecconf.org/proceedings/lrec2012/pdf/248_Paper.pdf.

Lars Borin, Maria Toporowska Gronostaj, and Dimitrios Kokkinakis. 2007. Medical frames as target and tool. In FRAME 2007: Building Frame Semantics Resources for Scandinavian and Baltic Languages. (Nodalida 2007 Workshop Proceedings). NEALT, Tartu, pages 11-18.

Aljoscha Burchardt, Katrin Erk, Anette Frank, Andrea Kowalski, Sebastian Padó, and Manfred Pinkal. 2009. Multilingual FrameNets in computational lexicography. Methods and Applications. Mouton de Gruyter, Berlin.

Marie Candito, Pascal Amsili, Lucie Barque, Farah Benamara, Gaël de Chalendar, Marianne Djemaa, Pauline Haas, Richard Huyghe, Yvette Yannick Mathieu, Philippe Muller, Benoît Sagot, and Laure Vieu. 2014. Developing a French framenet: Methodology and first results. In Proceedings LREC 2014. ELRA, Reykjavik, pages 1372-1379.

Oliver Christ. 1994. A modular and flexible architecture for an integrated corpus query system. ArXiv $\mathrm{abs} / \mathrm{cmp}-\mathrm{lg} / 9408005$.

Dana Dannélls, Lars Borin, Markus Forsberg, Karin Friberg Heppin, and Maria Toporowska Gronostaj. Forthc.a. Swedish FrameNet. In Dana Dannélls, Lars Borin, and Karin Friberg Heppin, editors, The Swedish FrameNet++: Harmonization, integration, method development and practical language technology applications, John Benjamins, Amsterdam, pages 37-65. Forthcoming in 2021.

Dana Dannélls, Lars Borin, and Karin Friberg Heppin, editors. Forthc.b. The Swedish FrameNet++: Harmonization, integration, method development and practical language technology applications. John Benjamins, Amsterdam. Forthcoming in 2021.

Christiane Fellbaum, editor. 1998. WordNet: An Electronic Lexical Database. MIT Press, Cambridge.

Charles J. Fillmore. 1977. Scenes-and-frames semantics. In Antonio Zampolli, editor, Linguistic Structures Processing, North Holland, Amsterdam, pages 55-81.

Charles J. Fillmore. 1982. Frame semantics. In Linguistic Society of Korea, editor, Linguistics in the Morning Calm, Hanshin Publishing Co., Seoul, pages 111-137.

Valentas Gruzauskas and Diwakaran Ragavan. 2020. Robotic process automation for document processing: A case study of a logistics service provider. Journal of Management Vol. 36:119-126. https://doi.org/10.38104/vadyba.2020.2.16.
Avi Hayoun and Michael Elhadad. 2016. The Hebrew FrameNet project. In Proceedings of LREC 2016. ELRA, Portorož, pages 4341-4347.

Jeong-uk Kim, Younggyun Hahm, and Key-Sun Choi. 2016. Korean FrameNet expansion based on projection of Japanese FrameNet. In Proceedings of COLING 2016, the 26th International Conference on Computational Linguistics: System Demonstrations. ACL, Osaka, pages 175-179.

Alessandro Lenci, Martina Johnson, and Gabriella Lapesa. 2010. Building an Italian FrameNet through semi-automatic corpus analysis. In Proceedings of LREC 2010. ELRA, Valletta. http://www.lrecconf.org/proceedings//rec2010/pdf/313_Paper.pdf.

Christopher D. Manning, Mihai Surdeanu, John Bauer, Jenny Finkel, Steven J. Bethard, and David McClosky. 2014. The Stanford CoreNLP natural language processing toolkit. In Proceedings of ACL 2014. ACL, Baltimore, pages 55-60. http://www.aclweb.org/anthology/P/P14/P14-5010.

Vasin Punyakanok, Dan Roth, and Wen tau Yih. 2008. The Importance of Syntactic Parsing and Inference in Semantic Role Labeling. Computational Linguistics 34(2).

Josef Ruppenhofer, Michael Ellsworth, Miriam R. L. Petruck, Christopher R. Johnson, Collin F. Baker, and Jan Scheffczyk. 2016. FrameNet II: Extended Theory and Practice. ICSI, Berkeley.

Hiroaki Saito, Shunta Kuboya, Takaaki Sone, Hayato Tagami, and Kyoko Ohara. 2008. The Japanese FrameNet software tools. In Proceedings of LREC 2008. ELRA, Marrakech.

Thomas C. Schmidt. 2009. The Kicktionary: A multilingual lexical resource of football language. In Hans C. Boas, editor, Multilingual FrameNets in Computational Lexicography. Methods and Applications, Mouton de Gruyter, Berlin, pages 101-134.

Carlos Subirats. 2009. Spanish Framenet: A framesemantic analysis of the spanish lexicon. In Hans C. Boas, editor, Multilingual FrameNets in Computational Lexicography. Methods and Applications, Mouton de Gruyter, Berlin, pages 135-162.

Tiago Timpioni Torrent. 2013. Behind the labels: Criteria for defining analytical categories in FrameNet Brasil. Veredas: Frame Semantics and Its Technological Applications 17(1):44-65.

Tiago Timponi Torrent, Maria Margarida Martins Salomão, Ely Edison da Silva Matos, Maucha Andrade Gamonal, Júlia Gonçalves, Bruno Pereira de Souza, Daniela Simões Gomes, and Simone Rodrigues Peron-Corrêa. 2014. Multilingual lexicographic annotation for domain-specific electronic dictionaries: The Copa 2014 FrameNet Brasil project. Constructions and Frames 6(1):73-91. 
Giulia Venturi. 2011. Semantic annotation of italian legal texts: A framenet-based approach. Constructions and Frames 3:46-79. https://doi.org/10.1075/cf.3.1.02ven.

Liping You, Tao Liu, and Kaiying Liu. 2007. Chinese Framenet and OWL representation. In Sixth International Conference on Advanced Language Processing and Web Information Technology (ALPIT 2007). pages 140-145. https://doi.org/10.1109/ALPIT.2007.50. 
A Developed Frames

\begin{tabular}{|c|c|c|c|}
\hline Frame & $\begin{array}{l}\text { Frame- } \\
\text { Elements }\end{array}$ & $\begin{array}{l}\text { Lexical } \\
\text { Units }\end{array}$ & Annotated Sentence \\
\hline Article & $\begin{array}{l}\text { Number, In- } \\
\text { terpretation, } \\
\text { Constitution, } \\
\text { Date }\end{array}$ & article.n & $\begin{array}{l}\text { The States Parties to the present Protocol under- } \\
\text { take to apply [articles]_LU [2 to } 34]_{-N u m b e r} \text { inclu- } \\
\text { sive of the [Convention to refugees]_Constitution as } \\
\text { hereinafter defined. }\end{array}$ \\
\hline Act & $\begin{array}{l}\text { Act, Purpose, } \\
\text { Conse- } \\
\text { quence, } \\
\text { Type }\end{array}$ & $\begin{array}{l}\text { act.n, act.v, } \\
\text { deed.n, } \\
\text { action.n }\end{array}$ & $\begin{array}{l}\text { Such violence must be given a broad interpre- } \\
\text { tation and may be defined as any [act]_LU of } \\
\text { [gender-based violence]_Act that results in, or is } \\
\text { likely to result in,[ physical, sexual or psycholog- } \\
\text { ical harm or suffering to women]_Consequence, in- } \\
\text { cluding threats of such acts, Confer/UNHCR003. }\end{array}$ \\
\hline Injunction & $\begin{array}{l}\text { Type, Injunc- } \\
\text { tion, Court, } \\
\text { Date }\end{array}$ & injunction.n & $\begin{array}{l}\text { In [July 2008]_Date the _ECHR, acting on a com- } \\
\text { plaint filed by the BHC, issued an [interim]_Type } \\
\text { [injunction]_LU to [halt the planned demolition of } \\
\text { Romani housing in Sofia]_Injunction. }\end{array}$ \\
\hline Judge & $\begin{array}{l}\text { Name, Des- } \\
\text { ignation, } \\
\text { Court }\end{array}$ & $\begin{array}{l}\text { judge.n, jus- } \\
\text { tice.n }\end{array}$ & $\begin{array}{l}\text { Justice_LU [Kirby]_Name similarly stated in the } \\
\text { same judgement that the Convention does not re- } \\
\text { quire or imply the elimination by the State of all } \\
\text { risks of harm; rather it "posits a reasonable level } \\
\text { of protection, not a perfect one". }\end{array}$ \\
\hline Judgement & $\begin{array}{l}\text { Judgement, } \\
\text { Type, Judge }\end{array}$ & judgement.n & $\begin{array}{l}\text { Justice Kirby similarly stated in the same } \\
\text { [judgement]]_LU that [the Convention does not } \\
\text { require or imply the elimination by the State of all } \\
\text { risks of harm; rather it "posits a reasonable level } \\
\text { of protection, not a perfect one"]]_Judgement. }\end{array}$ \\
\hline Defense & $\begin{array}{l}\text { Victim, } \\
\text { Defender, } \\
\text { Law, Type, } \\
\text { Place, Date, } \\
\text { Charges }\end{array}$ & $\begin{array}{l}\text { defence.n, } \\
\text { defend.v }\end{array}$ & $\begin{array}{l}\text { It is reasonable to conclude, therefore, that the } \\
\text { purpose of enacting section } 31 \text { was to meet the } \\
\text { difficulties exposed by the judgments in Adimi } \\
\text { by incorporating into domestic law, with cer- } \\
\text { tain modifications, the principles contained in } \\
\text { article } 31 \text { in the form of a [defence]_LU to [the } \\
\text { charges]_Charges most likely to be brought against } \\
\text { [asylum seekers]_Victim entering the country on } \\
\text { false passports. }\end{array}$ \\
\hline
\end{tabular}

